\title{
Malignant Skeletal Muscle Neoplasm
}

National Cancer Institute

\section{Source}

National Cancer Institute. Malignant Skeletal Muscle Neoplasm. NCI Thesaurus. Code C6516.

A malignant neoplasm arising from skeletal muscle. 